\title{
A Value-Oriented Price Forecasting Approach to Optimize Trading of Renewable Generation
}

\author{
Akylas Stratigakos \\ Center PERSEE \\ MINES ParisTech, PSL University \\ Sophia-Antipolis, France \\ akylas.stratigakos@mines-paristech.fr
}

\author{
Andrea Michiorri \\ Center PERSEE \\ MINES ParisTech, PSL University \\ Sophia-Antipolis, France \\ andrea.michiorri@mines-paristech.fr
}

\author{
Georges Kariniotakis \\ Center PERSEE \\ MINES ParisTech, PSL University \\ Sophia-Antipolis, France \\ georges.kariniotakis@mines-paristech.fr
}

\begin{abstract}
The participation of renewable generators in electricity markets involves employing a number of forecasting and decision-making tools. The standard approach consists in forecasting power output and market quantities, and then inputting the results into an optimization problem to derive optimal decisions. Typically, forecasting models are trained to optimize accuracy without considering the subsequent decisionmaking process. In this paper, we consider training forecasting models with a value-oriented approach that aims to minimize the suboptimality of decisions induced by a set of predicted inputs. We consider a risk-aware renewable generator participating in a day-ahead market subject to imbalance costs, and train ensembles of decision trees to forecast the imbalance penalty by directly minimizing trading costs for the provided strategy. The results indicate that our innovative approach leads to improved trading performance, compared to the standard method in which forecasting models are trained to minimize prediction errors.
\end{abstract}

Index Terms-Decision trees, electricity markets, energy trading, value-oriented forecasting, alternative loss functions.

\section{INTRODUCTION}

A plethora of problems related to power systems require making decisions under various forms of uncertainty [1]. The design of optimal participation strategies for renewable generators in competitive electricity markets is a prevalent example [2], since it usually requires solving some form of stochastic optimization problem, typically in a "Predict-then-Optimize" framework [3]. This involves first estimating an uncertain parameter at a future time interval, in a form that can range from deterministic (point) forecasts, to predictive densities, simulation trajectories and uncertainty sets. In a second step, these forecasts are used as input in an optimization problem.

When trading renewable energy on short-term markets, stochastic generation and market prices constitute typical sources of uncertainty. A significant body of work exists in this area, both for renewable energy [4] and electricity price [5] forecasting. Regarding price forecasting, most relevant work focuses on day-ahead (DA) prices. However, participation in

Parts of this research were carried in the frame of the European project REgions (No. 646039 and No. 775970), supported by ADEME's 'Investissement d'Avenir' program and the ERA-Net SES RegSys project, and the Smart4RES project (No. 864337), supported by the Horizon 2020 Framework Program.

978-1-6654-3597-0/21/\$31.00 @2021 IEEE multiple markets requires forecasting additional quantities, such as the imbalance penalty, defined as the difference between the DA price and the clearing price of the balancing market. A standard practice when forecasting the imbalance penalty is to split the problem into three components, namely direction and upward and downward regulation costs, and then model each one separately. In [6] an exponential smoothing approach is applied to capture the long-term trend and seasonality. A combination of a Seasonal ARIMA model with Markov Process is implemented in [7] to generate scenarios. In [8] a logistic regression model is employed to predict the imbalance direction, combined with ARIMA models for regulation costs.

A typical trading application would be a wind power plant (WPP) offering energy on the DA market subject to imbalance costs. In [9] the optimal risk-neutral offer is derived analytically as a quantile of the predictive density for wind generation, which also requires an estimation of the regulation costs. Extensions of this result include analytical expressions for the case of correlated DA and imbalance prices [10], considering adjustment markets [11], and jointly offering energy and primary [12] or secondary [13] reserve. The design of the balancing market is also an important factor, and particularly whether it features a single or dual price balancing mechanism [14]. Under a single price mechanism, any deviations from the submitted schedule that help restore system frequency result in additional revenue. For such cases, several risk-constrained trading strategies are proposed in [8].

In many cases, however, it is not clear how to move from a prediction to an optimal decision, as increased forecast accuracy does not always correspond to increased economic value [15]. It is therefore important to also assess forecast value [16], i.e., the impact of a forecast on decision costs, in addition to statistical accuracy. In this work, we aim to take this approach one step further by directly training a forecasting model to maximize value under a provided trading strategy.

Decision-making can be improved by jointly considering the forecasting and the optimization problem, and recent works combine elements from the fields of machine learning and operations research towards this goal. In [17] two data-driven approaches are proposed for directly solving the newsvendor problem, a form of direct policy selection. In [18], a general 
framework is proposed for using predictive machine learning algorithms to prescribe optimal decisions in the presence of explanatory data by solving a weighted Sample Average Approximation (SAA) of the original problem. The same work also introduces the coefficient of prescriptiveness, a unitless metric analogous to the $R^{2}$ coefficient in regression, which is used to assess the relative performance of prescribed decisions. From an applications standpoint, [19] proposes a data-driven approach to increase forecast accuracy and trading value in the presence of deterministic forecasts of wind power.

In the context of statistical learning, forecasting models are trained via loss minimization, with the objective of optimizing predictive accuracy or probabilistic performance. Training is performed without considering the subsequent optimization problem, which arises later on the model chain. Two valueoriented forecasting approaches are proposed in [20] for the case of a photovoltaic (PV) generator participating in the DA market. In the first, the individual energy and price forecasting models are tuned based on the decision costs, whereas in the second, a direct policy selection algorithm is applied, mapping the input data to decisions. Both approaches are formulated as non-linear optimization problems and approximated with evolutionary algorithms. In this work, we employ a generic, novel approach to training forecasting models in a supervised learning setting, known as Smart Predict-then-Optimize (SPO) [3]. A forecasting model is trained with the goal of generating predictions that prescribe optimal decisions. An alternative loss function, called SPO loss, is used to measure the suboptimality of decisions induced by a set of forecasted parameters. The proposed method can accommodate general multivariate and constrained optimization problems with a linear objective. Decision trees [21], including ensemble methods such as Random Forests, can be directly trained to minimize the SPO loss.

We consider the case of a virtual power plant (VPP), composed of wind and PV plants, participating in the DA market with a single price balancing mechanism. Assuming that the VPP generator has probabilistic forecasts for renewable generation at its disposal, we prescribe a profit-maximization strategy constrained in the probability space, which tunes the degree of risk-aversion. Based on this strategy, we derive the SPO loss function and train ensembles of decision trees to forecast the imbalance penalty for the DA horizon. We focus exclusively on imbalance penalties as they are very hard to predict, but the proposed framework is generic and can also accommodate energy forecasting models. We compare the efficacy of decisions to the standard approach, where a forecasting model is trained to minimize prediction error, and estimate a modified version of the coefficient of prescriptiveness. The results indicate that the proposed approach leads to improved decisions and increased forecast value.

The contribution of this work is threefold. First, we propose a novel approach for decision-making under uncertainty in electricity markets. Second, we derive the SPO loss function for a generic stochastic optimization problem, while previous works consider deterministic problems. Third, we advocate using the coefficient of prescriptiveness as an insightful measure of the relative efficacy compared to the base and perfectforesight solutions.

The remainder of the paper is organized as follows. Section II presents the SPO framework, Section III formulates the trading problem and Section IV presents the results. Finally, Section V concludes and provides directions for further research.

\section{THE SPO FRAMEWORK}

In this section, we provide a short description of the SPO framework and the training methodology, following [21]. For a nominal optimization problem with a linear objective, let $\omega \in \mathbb{R}^{d}$ denote the multivariate decision vector, $S$ the feasible set defined by convex constraints and $c \in \mathbb{R}^{d}$ the associated cost vector. Minimizing the objective function results in $z^{*}(c)=\min _{\omega \in S} c^{T} \omega$, with $\omega^{*}(c)=\operatorname{argmin}_{\omega \in S}\left\{c^{T} \omega\right\}$ being the optimal decision. Since $S$ is convex, $\omega^{*}(\cdot)$ is readily solved with off-the-shelf solvers.

In reality, the cost vector $c$ can be unknown. Instead, a training set of $n$ historical observations $\left\{\left(x_{1}, c_{1}\right), \cdots,\left(x_{n}, c_{n}\right)\right\}$ is available, where $x \in \mathbb{R}^{p}$ is a vector of $p$ explanatory features used to forecast $c$. A forecasting model $f(\cdot)$ maps observations of $x$ to $c$ and generates a predicted cost vector $\widehat{c}=f(x)$, which is subsequently used as input in the optimization problem, resulting in optimal decision $\omega^{*}(\widehat{c})$. During training, we search for an approximation $\widehat{f}(\cdot)$ by minimizing the value of a loss function $\ell(\widehat{c}, c)$ over the training data. For regression, the most common loss function is the Mean Square Error (MSE), defined as $\ell_{M S E}(\widehat{c}, c):=\|\widehat{c}-c\|_{2}^{2}$.

The SPO loss function [3] is used to directly train a forecasting model to minimize decision costs in the downstream optimization problem. For a predicted cost vector $\widehat{c}$ and an actual realization $c$, the SPO loss measures the difference in terms of cost as a result of taking decision $\omega^{*}(\widehat{c})$ instead of $\omega^{*}(c)$. For an optimization problem with a unique solution, the SPO loss function is defined as follows:

$$
\ell_{S P O}(\widehat{c}, c):=c^{T} \omega^{*}(\widehat{c})-z^{*}(c) .
$$

A value-oriented forecasting model thus uses (1) to approximate $f(\cdot)$. By applying the empirical risk minimization (ERM) principle, the following optimization problem is prescribed for training a forecasting model:

$$
f^{*}=\operatorname{argmin} \frac{1}{N} \sum_{i=1}^{N} \ell_{S P O}\left(f\left(x_{i}, c_{i}\right), c_{i}\right) .
$$

The SPO loss function can be non-convex and discontinuous, which poses challenges for training a machine learning algorithm. One approach proposed is to derive a surrogate loss function that provides an upper bound. A tractable methodology for directly training decision trees under the SPO loss is presented in [21].

Decision tree learning is a popular machine learning algorithm used both for regression and classification [22], as it offers intuitive and interpretable results. Decision trees work 
by recursively partitioning the feature space $\mathbb{R}^{p}$ at different components of $x$ using binary splits. Each split creates an internal node, with the final partitions being referred to as leaves of the tree. The predicted output for a new observation of $x$ that falls within a leaf equals the within-leaf mean or mode, for regression and classification respectively. The objective is to partition the feature space into $L$ leaves, $R_{1}, \ldots, R_{L}$, by minimizing:

$$
\min _{R_{1: L} \in T} \frac{1}{N} \sum_{l=1}^{L} \min _{\widehat{c}_{l}} \sum_{i \in R_{l}} \ell\left(\widehat{c}_{l}, c_{i}\right),
$$

where $T$ denotes the internal structure of the tree. The popular CART algorithm [23] is used to grow decision trees by "greedily" selecting locally optimal binary splits. Trees are typically grown large enough to capture non-linear dependencies among training data, and are then pruned back into a simpler version, to reduce overfitting.

The SPO Trees methodology suggests growing a decision tree following a CART-like approach that selects the split that leads to the greatest minimization of the SPO loss. The average cost vector $\bar{c}_{l}$ of all observations that fall within a leaf is the unique minimizer of the SPO loss within leaf $l$, as shown in [21]. This simplifies the task of training under the SPO loss and sets the basis for a tractable methodology of performing binary splits. The objective is:

$$
\min _{R_{1: L} \in T} \frac{1}{N} \sum_{l=1}^{L} \min _{\widehat{c}_{l}} \sum_{i \in R_{l}}\left(c_{i}^{T} \omega^{*}\left(\overline{c_{l}}\right)-z^{*}\left(c_{i}\right)\right) .
$$

The recursive partitioning approach of CART is applied with respect to (4) by evaluating the objective value at each candidate split and selecting the one that leads to lowest cost. For continuous features in $x$, a heuristic search is performed that only considers certain quantiles of the empirical distribution as candidate splits, in order to reduce the computational cost.

Decision trees are prone to overfitting, i.e., they are subject to high variance, which diminishes their predictive performance. Bootstrap aggregating (bagging) is a general methodology for reducing variance in estimators. First, $B$ new training sets are created by sampling with replacement (bootstrapping) from the original training set. Next, $B$ models are trained and individual predictions are aggregated via averaging or majority vote. The Random Forest algorithm [24] further expands on the bagging algorithm by training on de-correlated samples, using only a subset of the feature vector $x$ at each bootstrap iteration. The Random Forest algorithm significantly improves the predictive performance of decision trees. This methodology can be readily applied to SPO Trees to train ensembles of decision trees, which are called SPO Forests.

\section{RENEWABLE TRADING}

We proceed by formulating the problem of a renewable generator participating in the DA market under a single price balancing mechanism, focusing on the impact of imbalance penalty forecasts. Throughout, we assume that the generator is a price-taker, hence energy offers do not affect market prices, and probabilistic predictions for the renewable generation are available.

\section{A. Problem Formulation}

For a given delivery period $t$, let $\lambda^{D}$ denote the DA price and $\lambda^{B}$ the single clearing price of the balancing market. The imbalance penalty is defined as $\psi=\lambda^{D}-\lambda^{B}$. When the system is short, i.e., in need of upward regulation, it generally holds that $\lambda^{B} \geq \lambda^{D}$, therefore $\psi \leq 0$. The opposite is generally true when downward regulation is required, i.e., the system is long. However, these assumptions are regularly violated in practice, so we avoid imposing them moving forward.

Assuming energy quantities are normalized by the nominal capacity, the generator submits energy offers $E^{D} \in[0,1]$ for each delivery period $t$ of the next day. Given the absence of temporal constraints, optimization is conducted over each time period separately, thus subscript $t$ is dropped. The actual generation is a stochastic process denoted as $\tilde{E}$. The total revenue is also a stochastic process comprising two components, DA revenue and imbalance costs. Specifically:

$$
\tilde{\rho}=\lambda^{D} E^{D}+\lambda^{B}\left(\tilde{E}-E^{D}\right)=\lambda^{D} \tilde{E}-\psi\left(\tilde{E}-E^{D}\right) .
$$

From (5), we observe that when $\psi<0$, if the generator overproduces, i.e., $\tilde{E}>E^{D}$, the second term becomes positive and additional revenue is received. A risk-neutral participant maximizes revenue by solving the stochastic optimization problem $E^{D *}=\operatorname{argmin}(-\tilde{\rho})$. Under perfect foresight of $\psi$, the analytical solution to this problem prescribes offering either the nominal capacity or zero MWh [2]. Under uncertain market prices, the decision depends on the conditional expectation $\widehat{\psi}$ as:

$$
E^{D *}= \begin{cases}1, & \text { if } \widehat{\psi} \geq 0 \\ 0, & \text { if } \widehat{\psi}<0\end{cases}
$$

By examining (6) we observe that $E^{D *}$ depends only on the sign of the predicted penalty $\widehat{\psi}$, with $\widehat{\psi}=0$ being a trivial case. In reality, this strategy would expose participants to great risk due to large imbalances. In addition, the pricetaker assumption could be violated in practice. Constraining the optimal offer can lead to a better risk-return trade-off [8]. Assuming knowledge of the predictive cumulative density function (cdf) $\widehat{F}_{E}$ for the stochastic generation, we propose the following analytical rule for constraining the offer in the probability space:

$$
E^{D *}= \begin{cases}\widehat{q}_{1-a}, & \text { if } \widehat{\psi}>0 \\ \widehat{q}_{.50}, & \text { if } \widehat{\psi}=0 \\ \widehat{q}_{a}, & \text { if } \widehat{\psi}<0\end{cases}
$$

where $\widehat{q}_{a}$ is the $a$-quantile of $\widehat{F}_{E}$, with $a \in(0,0.50]$ being a design parameter that tunes the degree of risk-aversion. As $a \rightarrow 0.50$, the second term in (5), which corresponds to the energy imbalance, becomes smaller and the trading strategy becomes more risk-averse. The problem of identifying the 
optimal offer thus boils down to estimating the sign of the expectation $\widehat{\psi}$, which can be cast either as a regression or binary classification problem. Note that the cost of misclassification is dynamic, since it depends upon the absolute value of the realized penalties. Therefore, it is important to jointly consider direction and price in the forecasting model, as a wrong sign prediction in the presence of a large penalty would lead to excessive losses.

\section{B. Derivation of the SPO Loss Function}

Let $x^{i m b} \in \mathbb{R}^{p}$ denote the historic explanatory data used as input for predicting $\psi$. Given a prediction $\widehat{\psi}=f\left(x^{i m b}\right)$ and an actual realization $\psi$ of the imbalance penalty, we derive the optimal decisions from the analytical solution (7) as $E^{D *}(\widehat{\psi})$ and $E^{D *}(\psi)$, respectively. The revenue (5) remains a stochastic process, which depends on the realization of uncertain generation. One way to describe the SPO loss function is as the deviation from the expected revenue given uncertain renewable generation, which can be done either via sampling scenarios or by integrating over $\widehat{F}_{E}$. Alternatively, given the additional realization of actual generation $E^{o b s}$, we can define the SPO loss, in terms of costs, as follows:

$$
\ell_{S P O}(\hat{\psi}, \psi)=-\lambda^{D} E^{o b s}+\psi\left(E^{o b s}-E^{D *}(\hat{\psi})\right)+\rho^{*}(\psi),
$$

which finally results in:

$$
\ell_{S P O}(\hat{\psi}, \psi)=\psi\left(E^{D *}(\psi)-E^{D *}(\hat{\psi})\right) .
$$

From (9) we observe that if $\psi, \hat{\psi}$ have the same sign, no loss incurs. If the sign is different, then the loss equals the difference between $\widehat{q}_{a}$ and $\widehat{q}_{1-a}$, scaled by the imbalance penalty $\psi$. This means that the loss also depends on the dispersion of $\widehat{F}_{E}$. Observations that correspond to higher uncertainty in energy production, i.e., a larger dispersion in $\widehat{F}_{E}$, are thus weighted more heavily during training. This effect can be tuned by the selection of $a$. Overall, we observe that the training process is jointly affected by the sign and value of penalty $\psi$, the predictive density $\widehat{F}_{E}$, and the selected trading strategy.

\section{EVAluATION AND RESUlts}

\section{A. Case Study Description}

The proposed methodology is evaluated for the case of a VPP located in France, comprising wind and PV plants with a total capacity of $42.3 \mathrm{MW}$, and a $24 \%$ share of PV. Probabilistic predictions are obtained from [13] using a Quantile Regression Forest (QRF) model. Four months of probabilistic predictions at a 15-min resolution are available, with the first two used for training, and the rest for out-ofsample validation.

We used data from the German electricity market spanning the period April-July 2020. Explanatory data $x^{i m b}$ used for predicting $\psi$ comprise historical observations for imbalance price, system imbalance volume, DA price, and DA forecasts for system load, solar, wind and thermal generation. All market data are published prior to market closure and are available at [25]. In addition, one-hot encoded categorical variables are included to model possible calendar effects, namely day of the week, hour of the day and quarter of the hour. Since the DA market closes at 9h00 UTC on the previous day, the clearing price for the balancing market and the system imbalance volume of that day are unavailable. Instead, historical observations from two days prior to the delivery are used, as well as the value last observed at the forecast origin.

\section{B. Benchmarks}

Two additional price forecasting models are trained under the standard Predict-then-Optimize framework and evaluated based on trading results obtained by applying the riskconstrained strategy described in (7). In order to better illustrate the added value of training under the SPO loss instead of a traditional loss function, two variations of the Random Forest algorithm are considered. Specifically, the benchmarks are:

- Random Forest Regressor (RF-Reg): A Random Forest model is trained in a typical regression fashion to predict $\psi$ by minimizing MSE loss during training.

- Random Forest Classifier (RF-Class): A Random Forest classifier is trained to predict the probability $\phi$ of the system being short. Here, splits are decided based on the reduction of the Gini index, which measures the impurity of each node [22]. Following (7), if $\widehat{\phi}>0.5$, then the optimal offer is $\widehat{q}_{a}$, or else $\widehat{q}_{1-a}$.

Note that the decision threshold for the RF-Class model is set naively at 0.5 , without considering any asymmetry in imbalance penalties. However, tuning for this threshold requires an ad hoc estimation of the value of imbalance penalties, which itself is a regression problem. As our goal is to showcase improved performance of a single model that uses an alternative loss function, we avoid developing a separate model to tune this parameter. Finally, in order to present more comprehensive results, the Expected Value $(E V)$ offering strategy is also evaluated, with the offer being $E^{D *}=\mathbb{E}\left(\widehat{F}_{E}\right)$. The EV strategy depends only on renewable generation forecasts and provides a trading benchmark.

Preliminary analysis indicated that modeling each 15-min interval separately does not offer added value, thus a single model is trained for all time steps. All trees are fully grown without pruning. Ensemble tree algorithms are considered fairly robust against overfitting and immune to noisy inputs, and thus do not require extensive feature selection. The number of trees $B$ to grow is set at 300 throughout, which is adequate to capture non-linear dependencies and ensure convergence of the ensemble effect. Two hyperparameters require further tuning, namely the size $m$ of the feature subset selected at each bootstrap iteration and the minimum number $n_{\text {min }}$ of sample observations required to split an internal node, which is conducted via grid-search with a 5-fold crossvalidation. For the SPO Forests in particular, hyperparameter tuning is conducted using a custom scoring rule that evaluates the expected revenue during cross-validation. Moreover, for the SPO Forests a different model is trained for each value of the risk-parameter $a$ in (7) and continuous features of 
TABLE I

Trading Results for the Testing Period

\begin{tabular}{|c|c|c|c|c|c|c|c|c|c|c|}
\hline \multirow{2}{*}{$\begin{array}{l}\text { Trading } \\
\text { Results }\end{array}$} & \multirow[b]{2}{*}{ EV } & \multicolumn{3}{|c|}{$a=0.05$} & \multicolumn{3}{|c|}{$a=0.10$} & \multicolumn{3}{|c|}{$a=0.20$} \\
\hline & & SPO Forest & RF-Reg & RF-Class & SPO Forest & RF-Reg & RF-Class & SPO Forest & RF-Reg & RF-Class \\
\hline Total revenue (EUR) & 354674 & 369121 & 367395 & 355893 & 370808 & 366656 & 357700 & 363380 & 362946 & 357241 \\
\hline$C V a R_{5 \%}($ EUR $)$ & -78.02 & -141.50 & -167.80 & -178.51 & -111.63 & -129.75 & -139.34 & -88.11 & -101.15 & -107.01 \\
\hline Energy bid (MWh) & 9677 & 6902 & 10310 & 9626 & 7432 & 10090 & 9562 & 8123 & 9914 & 9567 \\
\hline $\begin{array}{c}\text { Absolute bid } \\
\text { deviation }(\mathrm{MWh})\end{array}$ & 2676 & 5121 & 5173 & 5138 & 4343 & 4299 & 4283 & 3529 & 3458 & 3461 \\
\hline $\begin{array}{c}\text { Revenue per } \\
\text { imbalance (EUR/MWh) }\end{array}$ & 0.85 & 3.26 & 2.90 & 0.68 & 4.24 & 3.32 & 1.24 & 3.11 & 3.05 & 1.40 \\
\hline
\end{tabular}

$x^{i m b}$ are evaluated at quantiles $\{0.10, \ldots, 0.90\}$. The SPO Forests are trained using the implementation provided at https://github.com/rtm2130/SPOTree.

\section{Results}

We assess the out-of-sample trading performance of the forecasting models over the 2-month testing period. The $5 \%$ Conditional Value at Risk $\left(\mathrm{CVaR} \mathrm{R}_{5 \%}\right)$, defined as the expected profit in the worst $5 \%$ of cases, is used to measure trading risk. Table I presents analytical results for $a=\{0.05,0.10,0.20\}$. Overall, the SPO Forest model outperforms the other benchmarks in terms of revenue for a given value of $a$, reaching up to a $4.5 \%$ increase compared to the EV strategy for $a=0.10$. In general, lower values of $a$ lead to an increase in both revenue and risk, as evidenced by a decrease in $C V a R_{5 \%}$, which is in accordance with our expectations for a riskaware trading strategy. This fact is highlighted in Fig. 1, where aggregated revenue is plotted against $C V a R_{5} \%$ for the different levels of $a$. Fig. 1 shows the SPO Forest model leads to improved overall performance, both in terms of generated revenue and risk, with consistent results across the different degrees of risk-aversion. Specifically, the line that corresponds to the SPO Forests sets the efficient frontier, i.e., for a given amount of risk the SPO Forests result in the largest revenue across the different models (and vice versa). As $a \rightarrow 0.50$, all models converge to a similar performance. The RF-Class model performs the worst overall, offering only a marginal revenue increase compared to the EV strategy, accompanied by a large increase in risk.

An interesting result observed from Table I is that the SPO Forest model results in the VPP offering the least amount of energy in the DA market across the different values of $a$. This could be attributed to the SPO Forest model adapting to the asymmetry between the upward and downward regulation costs. Indeed, the in-sample absolute averages for upward and downward penalties are approximately 40 EUR/MWh and 37 EUR/MWh, respectively, which probably causes the SPO Forest model to favor offers below the expected generation. We further observe that all models achieve a positive revenue per imbalance. This is attributed to the single price balancing mechanism, but is also highly dependent on the selected testing set. For example, the fact that the EV strategy achieves a positive revenue per imbalance is purely coincidental, since

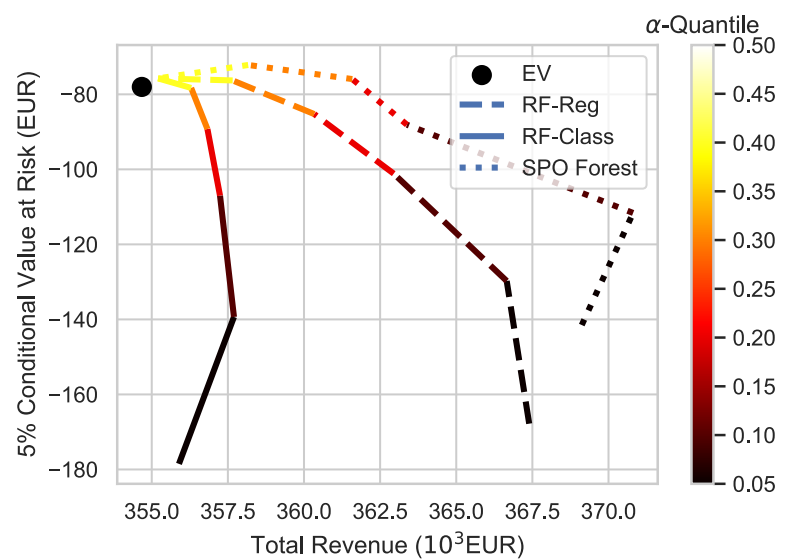

Fig. 1. Risk vs Total Revenue for different values of $a$

in this case imbalances only depend on generation forecast errors.

We further examine the relative efficacy of the prescribed decisions by estimating a modified version of the coefficient of prescriptiveness $P$ [18]. For each model $i$ and each value of risk-parameter $a$, we estimate the respective coefficient $P$ as:

$$
P_{i, a}=1-\frac{\widehat{\rho}_{i, a}-\rho_{a}^{*}}{\widehat{\rho}_{E V}-\rho_{a}^{*}},
$$

where $\rho_{a}^{*}$ is the revenue obtained by the deterministic perfectforesight strategy, i.e., assuming perfect knowledge of $\psi$, following the strategy prescribed in (7), and $\widehat{\rho}_{E V}$, the revenue obtained by the EV strategy. Coefficient $P$ is bounded above by 1 and can be interpreted as a measure of the relative "distance", defined strictly in terms of revenue, between the benchmark EV strategy and the theoretical maximum. Fig. 2 shows the evolution of $P$ for the different forecasting models and different values of $a$ (for $a=0.50, P=1$ in all cases). As expected, the SPO Forest model shows improved relative performance, followed by the RF-Reg model, with the difference being more pronounced for $a=0.10$. The RFClass model performs the worst overall. However, it should be noted that the coefficient $P$ is generally low in all cases, with a maximum value of just over 0.10 . This means that, even in the best case, the prescribed decisions are still relatively far from obtaining maximum revenue. This fact highlights the 


\section{REFERENCES}

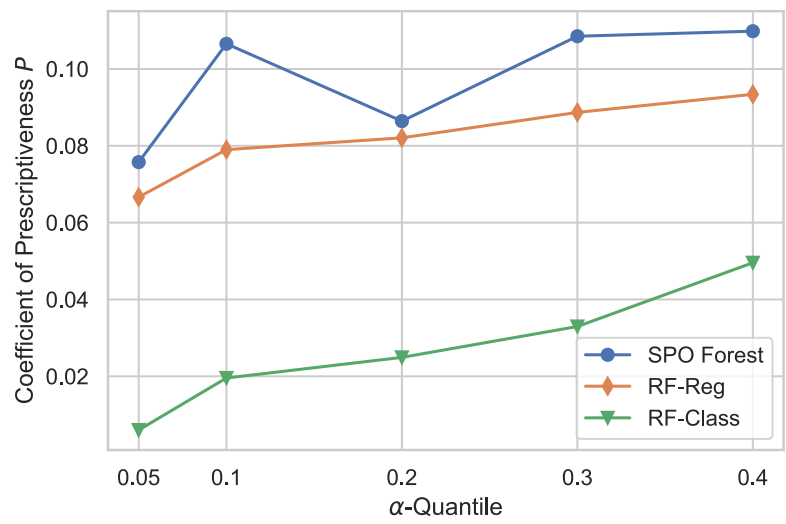

Fig. 2. Estimated coefficient of prescriptiveness per risk level $a$

inherent difficulty in forecasting the imbalance penalty on the DA horizon, which in turn imposes practical limitations to exploiting the single price balancing mechanism for additional revenue.

\section{CONCLUSIONS}

In this work, we proposed a value-oriented forecasting approach for prescribing optimal decisions for trading renewable energy. This involved training a forecasting model taking into consideration the subsequent optimization problem, using the SPO loss function to measure excess cost due to erroneous predictions.

We considered the case of a VPP generator offering energy in a DA market under a single price balancing mechanism and employed a profit maximization strategy constrained in the probability space. Ensembles of decision trees were trained to forecast the imbalance penalty by minimizing the SPO loss function and applying the Random Forest algorithm. In addition, we estimated the coefficient of prescriptiveness for the different models and evaluated relative performance against the benchmark and the perfect-foresight strategy. Overall, results indicate that for a given trading strategy, using a forecasting model trained under the SPO loss function leads to better decision-making and improved performance, both in terms of revenue and risk. Moreover, the proposed approach offers an attractive alternative to the benchmark trading strategy. In future work, we aim at applying the proposed methodology in more complex case studies, such as jointly considering energy and market price forecasting, and assessing the impact of explanatory variables in trading performance.

\section{ACKNOWLEDGMENT}

The authors would like to thank HESPUL and ENGIE GREEN for contributing historical production data, Simon Camal for providing historical probabilistic forecasts and Panagiotis Andrianesis for his insightful comments.
[1] W. B. Powell and S. Meisel, "Tutorial on stochastic optimization in energy - part i: Modeling and policies," IEEE Transactions on Power Systems, vol. 31, no. 2, pp. 1459-1467, 2015.

[2] J. M. Morales, A. J. Conejo, H. Madsen, P. Pinson, and M. Zugno, Integrating renewables in electricity markets: operational problems. Springer Science \& Business Media, 2013, vol. 205.

[3] A. N. Elmachtoub and P. Grigas, "Smart" predict, then optimize"," arXiv preprint arXiv:1710.08005, 2017.

[4] G. Kariniotakis, Renewable Energy Forecasting: From Models to Applications. Woodhead Publishing, 2017.

[5] R. Weron, "Electricity price forecasting: A review of the state-of-the-art with a look into the future," International journal of forecasting, vol. 30, no. 4, pp. 1030-1081, 2014.

[6] T. Jónsson, P. Pinson, H. A. Nielsen, and H. Madsen, "Exponential smoothing approaches for prediction in real-time electricity markets," Energies, vol. 7, no. 6, pp. 3710-3732, 2014.

[7] M. Olsson and L. Soder, "Modeling real-time balancing power market prices using combined sarima and markov processes," IEEE Transactions on Power Systems, vol. 23, no. 2, pp. 443-450, 2008.

[8] J. Browell, "Risk constrained trading strategies for stochastic generation with a single-price balancing market," Energies, vol. 11, no. 6, p. 1345 , 2018.

[9] P. Pinson, C. Chevallier, and G. N. Kariniotakis, "Trading wind generation from short-term probabilistic forecasts of wind power," IEEE Transactions on Power Systems, vol. 22, no. 3, pp. 1148-1156, 2007.

[10] C. J. Dent, J. W. Bialek, and B. F. Hobbs, "Opportunity cost bidding by wind generators in forward markets: Analytical results," IEEE Transactions on Power Systems, vol. 26, no. 3, pp. 1600-1608, 2011.

[11] J. M. Morales, A. J. Conejo, and J. Pérez-Ruiz, "Short-term trading for a wind power producer," IEEE Transactions on Power Systems, vol. 25 , no. 1, pp. 554-564, 2010.

[12] J. Liang, S. Grijalva, and R. G. Harley, "Increased wind revenue and system security by trading wind power in energy and regulation reserve markets," IEEE Transactions on Sustainable Energy, vol. 2, no. 3, pp. 340-347, 2011.

[13] S. Camal, A. Michiorri, and G. Kariniotakis, "Optimal offer of automatic frequency restoration reserve from a combined pv/wind virtual power plant," IEEE Transactions on Power Systems, vol. 33, no. 6, pp. 61556170, 2018.

[14] ENTSO-E, "Survey on ancillary services procurement, balancing market design," 2018

[15] H. Zareipour, C. A. Canizares, and K. Bhattacharya, "Economic impact of electricity market price forecasting errors: A demand-side analysis," IEEE Transactions on Power Systems, vol. 25, no. 1, pp. 254-262, 2009.

[16] C. Kath and F. Ziel, "The value of forecasts: Quantifying the economic gains of accurate quarter-hourly electricity price forecasts," Energy Economics, vol. 76, pp. 411-423, 2018.

[17] G.-Y. Ban and C. Rudin, "The big data newsvendor: Practical insights from machine learning," Operations Research, vol. 67, no. 1, pp. 90$108,2019$.

[18] D. Bertsimas and N. Kallus, "From predictive to prescriptive analytics," Management Science, vol. 66, no. 3, pp. 1025-1044, 2020.

[19] M. A. Munoz, J. M. Morales, and S. Pineda, "Feature-driven improvement of renewable energy forecasting and trading," IEEE Transactions on Power Systems, 2020.

[20] T. Carriere and G. Kariniotakis, "An Integrated Approach for ValueOriented Energy Forecasting and Data-Driven Decision-Making Application to Renewable Energy Trading," IEEE Transactions on Smart Grid, vol. 10, no. 6, pp. 6933-6944, 2019.

[21] A. N. Elmachtoub, J. C. N. Liang, and R. McNellis, "Decision trees for decision-making under the predict-then-optimize framework," arXiv preprint arXiv:2003.00360, 2020.

[22] T. Hastie, R. Tibshirani, and J. Friedman, The elements of statistical learning: data mining, inference, and prediction. Springer Science \& Business Media, 2009.

[23] L. Breiman, J. Friedman, C. J. Stone, and R. A. Olshen, Classification and regression trees. CRC press, 1984.

[24] L. Breiman, "Random forests," Machine learning, vol. 45, no. 1, pp 5-32, 2001.

[25] ENTSO-E. Transparency platform. [Online]. Available: https:// transparency.entsoe.eu/ 